\title{
Distributional Properties of Relative Phase in Bimanual Coordination
}

\author{
Eric James, Charles S. Layne, Karl M. Newell
}

\begin{abstract}
Studies of bimanual coordination have typically estimated the stability of coordination patterns through the use of the circular standard deviation of relative phase. The interpretation of this statistic depends upon the assumption of a von Mises distribution. The present study tested this assumption by examining the distributional properties of relative phase in three bimanual coordination patterns. There were significant deviations from the von Mises distribution due to differences in the kurtosis of distributions. The kurtosis depended upon the relative phase pattern performed, with leptokurtic distributions occurring in the in-phase and antiphase patterns and platykurtic distributions occurring in the $30^{\circ}$ pattern. Thus, the distributional assumptions needed to validly and reliably use the standard deviation are not necessarily present in relative phase data though they are qualitatively consistent with the landscape properties of the intrinsic dynamics.
\end{abstract}

Keywords: coordination dynamics, distributional properties, relative phase

In the synergetic approach to the study of dynamic systems (Haken, 1977, 1984) an experimentally identified order parameter describes a consistent and coherent pattern of the components of a system. Control parameters, that need not be specific to the order parameter, lead the system through its possible states as captured by the order parameter. Kelso (1984) identified the presence of phase transitions in the relative phase of bimanual coordination, thereby supporting the postulation of relative phase as the order parameter in bimanual coordination and movement frequency as a control parameter (Haken, Kelso, \& Bunz, 1985; Kelso, 1995).

The standard deviation of relative phase has been a typical means of quantifying the stability of this order parameter in bimanual coordination. The Haken, Kelso and Bunz model (HKB model; 1985) captures the stability properties of bimanual coordination patterns and the occurrence of phase transitions with the scaling of a control parameter (movement frequency). Subsequent experiments have also identified the presence of additional phenomena in bimanual coordination such as critical fluctuations, hysteresis and critical slowing down that are hallmarks of the behavior of dynamic systems (Byblow, Summers, Semjen, Wuyts, \& Carson, 1999; Post, Peper, Daffertshofer, \& Beek, 2000; Scholz \& Kelso, 1990).

James is with the Department of Health and Human Performance, University of Texas, Brownsville, TX. Layne is with the Laboratory of Integrated Physiology, University of Houston, Houston, TX. Newell is with the Department of Kinesiology, The Pennsylvania State University, University Park, PA. 
Schöner, Haken and Kelso (1986) introduced a stochastic term to the original HKB model to represent perturbations to relative phase during the performance of bimanual coordination patterns. The equation for the SHK model is:

$$
\frac{d \varphi}{d t}=-a \sin (\varphi)-2 b \sin (2 \varphi)+\sqrt{Q} \xi
$$

Where $\varphi$ is relative phase, $a$ and $b$ are control parameters, $\xi$ is white Gaussian noise and $Q$ is a parameter for the strength of this noise. With the inclusion of the stochastic term the model captures fluctuations in relative phase. These fluctuations induce phase transitions from antiphase to in-phase at control parameter values at which a limited degree of stability remains within the antiphase pattern. Empirical evidence has supported this model with respect to changes in the SD/variance of relative phase fluctuations at different control parameter values (Schöner et al., 1986) as well as the control parameter values at which phase transitions occur (Molenaar \& Newell, 2003).

Recently, it has been shown that the fluctuations in relative phase time series are not noise but contain time-dependent structure (Torre, Delignières, \& Lemoine, 2007). The finding of time dependent structure within fluctuations of the relative phase of bimanual coordination is consistent with a number of prior studies that have found that the variability of motor output typically does not follow a distribution of white Gaussian noise (see Newell \& Slifkin, 1998 for a review). For example, structure within the time domain have been found in the variability of discrete movements (Gilden, Thornton, \& Mallon, 1995), continuous force production (Slifkin \& Newell, 1999) and in fluctuations of the postural center of pressure (Yamada, 1995). The variability of motor output has also been found to deviate from the skewness and kurtosis of a Gaussian distribution (Newell \& Hancock, 1984).

Lee, Blandin and Proteau (1996) have previously reported descriptive statistics of positive (i.e., leptokurtic) distributions in the in-phase and antiphase patterns at low movement frequencies. They found that kurtosis decreased as movement frequency increased, and even became negative (i.e., leptokurtic) in the antiphase pattern at high movement frequencies. However, in this study an inferential analysis of kurtosis data were not performed and the kurtosis values calculated were linear, rather than circular, even though relative phase is a circular variable. In addition, the point estimate of relative phase was calculated for only $12 \mathrm{~s}$ of data in each trial, making an estimation of the 4th distributional moment of data potentially unreliable.

When characterizing the variability of bimanual coordination with the $S D$ of relative phase a general assumption is that the data follow a Gaussian distribution. The distribution of relative phase data in bimanual coordination has been described as violating the assumption of normality (Lee et al., 1996) but this assumption has not been formally tested. Violations of this assumption may invalidate statistical inferences and the comparison of standard deviation, mean, median and mode across groups (Fisher, 1930).

While the use of the $S D$ to estimate variability assumes the presence of a Gaussian distribution estimates of the degree of entropy make no assumption regarding distributional properties. In the calculation of entropy the potential range of data are divided into bins. An estimate of entropy is calculated based upon the probability of data occurring within each bin. In a broad distribution with an approximately 
equal number of occurrences in each bin is high in entropy (and variability). When data are clustered within a few bins the entropy is low (Williams, 1997).

As the calculation and interpretation of estimates of entropy do not depend upon the presence of a Gaussian distribution this type of statistic is more versatile than the $S D$ and can be used to characterize the amount of variability within more types of data. For example, in the analysis of relative phase variability it can be inappropriate to use the $S D$ to estimate variability in intrinsically unstable coordination patterns that may not be statistically differentiable from a uniform distribution. Entropy is a statistically appropriate means of estimating variability in such cases.

A potential danger in using the $S D$ to characterize variability in non-Gaussian distributions of data is that inaccurate conclusions might be drawn when comparing the variability of different data sets. Differences in the $S D$ of different sets of data might be attributed to differences in variability while these may in fact only be due to differences in the distributional properties of the sets of data (Newell \& Hancock, 1984). This type of erroneous conclusion can be avoided by using entropy to estimate variability, as this type of statistic is independent of the distributional properties of data (Williams, 1997).

In addition, a theoretical complication of the HKB model and its derivations is an inconsistency between the assumptions of the term $\xi$ as white Gaussian noise and the parameter relative phase. Relative phase is a circular variable in which, for example, a value of 0 radians (alternatively $0^{\circ}$ ) is the same as that of $2 \pi$ radians (alternatively $360^{\circ}$ ). However, in linear statistics these two values are not equivalent. A Gaussian distribution, on the other hand, pertains to linear variables. Low data values within the linear Gaussian distribution cannot be equivalent to high values, as can occur with relative phase and circular statistics in general. In standard circular statistics the counterpart to the Gaussian distribution is the von Mises distribution. The properties of a von Mises distribution, which possesses a skewness and kurtosis of zero, are calculated using the sine and cosine functions. Circular inferential and descriptive statistics assume the presence of this distribution (Batschelet, 1981).

In the present experiment we examined the von Mises distribution to investigate the inconsistency between the Gaussian $\xi$ term in the SHK model and relative phase, and to allow an experimental analysis of the distributional properties of the circular variable relative phase. If relative phase fluctuations do not follow a von Mises distribution then the traditional analysis of relative phase variability and stability through the use of the circular $S D$ may not be appropriate. Therefore, if the higher moment distributional properties within data are not held constant a comparison of conditions based on the first and second moments of the distribution (i.e., the mean and $S D$ ) can be invalid (Fisher, 1930).

\section{Methods}

\section{Participants}

Young adult participants $(N=14 ; M=22.4$ years; $S D=3.1$ years $)$ were recruited from the University of Houston student body. All participants provided informed consent, and all procedures were in accordance with guidelines of the Institutional Review Board of the University. 


\section{Apparatus}

Participants had a custom made cylindrical cardboard sleeve placed over each forearm and hand, grasping a wooden handle inside the distal end of each sleeve. These cylindrical sleeves were of dimension $35 \mathrm{~cm}$ (length) $\times 7.5 \mathrm{~cm}$ (diameter), weight $121 \mathrm{~g}$ each and were held in place by two elastic and Velcro straps. Two infrared-emitting diodes were placed on the distal surface of each sleeve, the movements of which were tracked in three dimensions by an Optotrak 3020 (Northern Digital Inc.; Waterloo, Ontario) motion analysis system. These cylindrical sleeves were used to prevent the recruitment of the wrist as a degree of freedom in the movement and also served as a mount for the infrared LEDs that were tracked by the Optotrak system. These sleeves are depicted in Figure 1.

The Optotrak system has a root mean square accuracy of $0.1 \mathrm{~mm}$ for $x$ - and $y$ - coordinates, at a distance of $2.25 \mathrm{~m}$, and of $0.15 \mathrm{~mm}$ for the $z$ - coordinate at the same distance (Northern Digital Inc. web site). The Optotrak system software was run on a Dell Dimension 4550 computer with a sampling rate of $200 \mathrm{~Hz}$. A Test Tone Generator auditory metronome with adjustable phase and tone duration settings was used to provide auditory tones and was run on an Acer Aspire 3003LCi laptop computer placed alongside each participant. The phase setting on this auditory metronome was set to match trial relative phase conditions and a 100 ms tone was used in all trials.

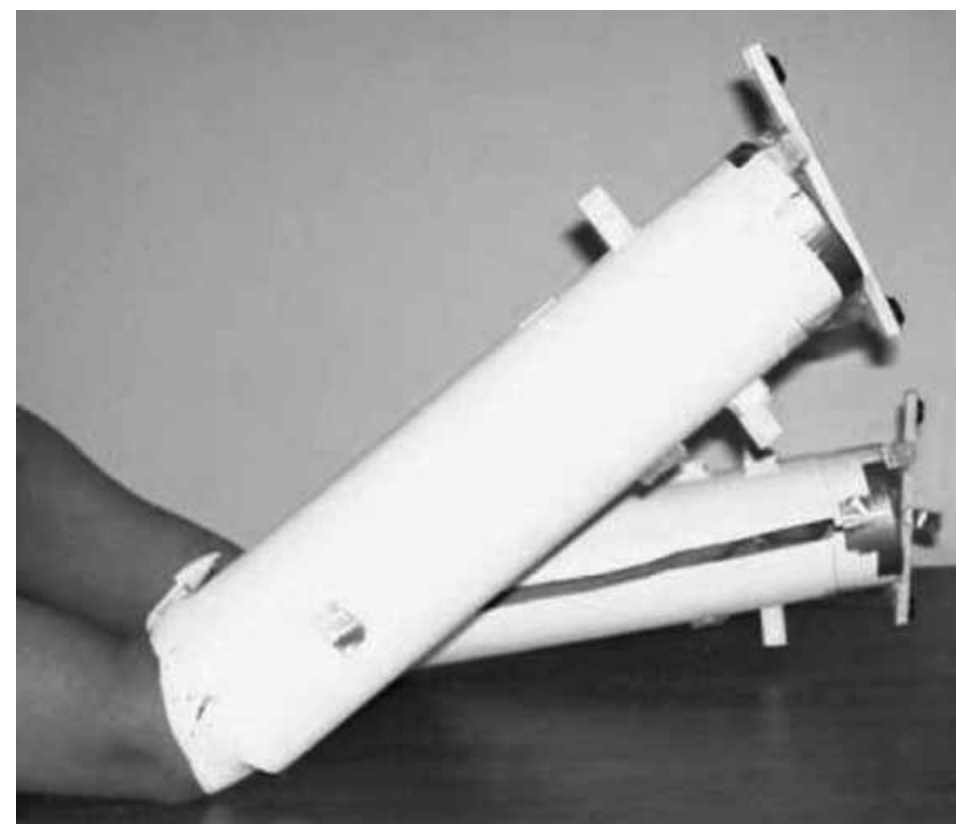

Figure 1 - The cylindrical sleeves worn over the forearms, wrists and hands to eliminate the recruitment of movement at the wrist joints. 


\section{Procedure}

Participants were seated $3 \mathrm{~m}$ in front of and facing the Optotrak 3020 system with elbows resting on a padded tabletop and upper arms at approximately a $45^{\circ}$ angle. Participant elbows did not move during performance and were not restrained. A computer generated auditory metronome was played for $10 \mathrm{~s}$ immediately before and during movement trial data collection.

The task consisted of making flexion-extension movements of the elbow joints through approximately $90^{\circ}$ of motion with the points of maximum extension of each arm in synchronization with the computer generated auditory tones. Participants performed three bimanual coordination patterns: in-phase, antiphase and $30^{\circ}$ relative phase. In each relative phase condition auditory tones were generated such that by alternately synchronizing the points of maximum elbow extension with consecutive tones the appropriate relative phase for each condition would be produced at a frequency of $1.1 \mathrm{~Hz}$. In the in-phase condition the computer generated auditory tones were equally spaced at a frequency of $1.1 \mathrm{~Hz}$ rate with participants moving both arms together. In the antiphase condition the auditory tones were spaced with a $180^{\circ}$ degree phase lag, which effectively consisted of tones played at a frequency of $0.55 \mathrm{~Hz}$, but with alternate elbow extension coinciding with alternate auditory tones. In the $30^{\circ}$ relative phase condition tones were phase lagged such that by coinciding alternate points of maximum arm extension with alternate auditory tones this relative phase pattern would be produced.

One trial of $180 \mathrm{~s}$ duration was performed in each relative phase condition with condition order counterbalanced across participants. Participants were instructed to begin moving their arms in the required fashion as soon as the auditory pacing tones began and data collection was initiated $10 \mathrm{~s}$ after the auditory tones began.

\section{Data Analyses}

Kinematic data for each $180 \mathrm{~s}$ movement trial were recorded by the Optotrak system at a sampling rate of $200 \mathrm{~Hz}$, yielding time series of 16000 data points for each infrared light emitting diode marker and directional axis. Data pertaining to movement of the four infrared light emitting diode markers in the $y-z$ (saggital) plane were filtered with a 4th order $10 \mathrm{~Hz}$ low-pass Butterworth filter and were used to determine the angular position of each arm at each time point.

In bimanual coordination the estimation of relative phase, which is considered the order parameter of the system, has been performed via several methods. One method is the Point Estimate of Relative Phase (PRP; Kelso, 1995). This method uses the equation:

$$
P R P=\frac{\left(t_{1}-t_{2}\right)}{T} \times 360^{\circ}
$$

where $t_{1}$ and $t_{2}$ are times of local maximum (peak extension) for the two forearms and $T$ is the period (time between consecutive $t_{1}$ occurrences). The use of PRP allowed for the analysis of across-cycle fluctuations in relative phase and was 
consistent with previous research on the properties of fluctuations in relative phase (Torre et al., 2007).

The PRP method was used to calculate the first 180 consecutive PRP values of both the maximum and minimum positional values (i.e., maximum elbow extension and flexion). In this way previously identified issues with continuous relative phase (Torre et al., 2007) were avoided while still providing information regarding fluctuations in relative phase at two different points during movement cycles. In the results section the term maximum PRP denotes relative phase at points of maximum extension (at which synchronization with auditory tones occurred) while the term minimum PRP denotes relative phase at points of minimum extension at which no auditory tones occurred.

The information entropy (Shannon, 1948), $H$, of relative phase was calculated as:

$$
H=-\Sigma p_{i} \log _{2} p_{i}
$$

where $p_{i}$ is the probability of data points occurring within the $i^{\text {th }}$ bin, with each bin being $10^{\circ}$ in width with a total of 36 bins. Low information entropy values indicate a probability distribution concentrated within relatively few bins and low variability while high entropy values indicate a more equal distribution of data across bins and a greater degree of variability within the distribution.

As relative phase is a circular variable standard circular statistics were used in its analysis (Batschelet, 1981). The Raleigh uniformity test was used to determine if the relative phase of each time series was significantly clustered around a modal value. A uniform distribution of relative phase values within a time series would indicate that the participant did not produce a statistically identifiable coordination pattern. Relative phase time series identified as uniformly distributed were removed from subsequent analysis.

Watson's $\mathrm{U}^{2}$ test (Batschelet, 1981) was used to determine if the relative phase time series differed significantly from a von Mises distribution. This test compares the goodness-of-fit of experimental data with the von Mises distribution. The mean squared deviation between the experimental and von Mises distributions is calculated. When this deviation is sufficiently high the experimental data are shown to be significantly different than the von Mises distribution.

For time series found to differ significantly from a von Mises distribution statistical tests of the skewness and kurtosis were conducted to determine the manner of deviation from this distribution. One-sample $t$ tests of means were conducted on the circular skewness and kurtosis data to determine if these 3rd and 4th order statistical moments of experimental relative phase time series differed from those of a von Mises distribution. A 3 (Relative Phase) $\times 2$ (Min/Max Endpoint) ANOVA of relative phase kurtosis values was performed to determine if this dependent variable changed as a function of these relative phase conditions or the endpoint of bimanual movements, one of which (minimum PRP) was synchronized with auditory tones.

In all analyses a type I error of $\alpha=.05$ was used to determine statistical significance. The calculation of dependent variable values was performed with coded MATLAB (Mathworks, Natick, MA) programs. All circular statistical analyses were performed in Oriana (Anglesey, Wales) circular statistics software. 


\section{Results}

\section{Uniformity}

Rayleigh tests of uniformity showed that out of 84 total trials $75(89.29 \%)$ differed significantly from a uniform distribution. These nonuniform trials consisted of: 13 trials (out of 14; 92.86\%) in the in-phase minimum PRP condition, 14 trials $(100 \%)$ in-phase maximum PRP; 13 trials $(92.86 \%)$ in the antiphase minimum PRP condition, 14 trials $(100 \%)$ in the antiphase maximum PRP condition; 10 trials $(71.43 \%)$ in the $30^{\circ}$ minimum PRP condition, and 11 trials $(78.57 \%)$ in the $30^{\circ}$ maximum PRP.

\section{von Mises Distribution}

After the uniformly distributed trials were removed from further analyses, Watson's $\mathrm{U}^{2}$ tests indicated that a total of 42 trials (out of $75 ; 56 \%$ ) differed significantly from a von Mises distribution. The trials that did not follow a von Mises distribution consisted of 13 trials $(100 \%)$ in the in-phase minimum PRP condition, 12 trials $(85.71 \%)$ in the in-phase maximum PRP condition, 6 trials $(46.15 \%)$ in the antiphase minimum PRP condition, 3 trials $(21.43 \%)$ in the maximum PRP condition, 3 trials $(30 \%)$ in the $30^{\circ}$ minimum PRP condition, and 5 trials $(45.45 \%)$ in the $30^{\circ}$ maximum PRP condition.

\section{Entropy}

As shown in Figure 2 the entropy in the in-phase minimum PRP condition was $1.76(S D=1.01)$ and for the maximum PRP condition $1.55(S D=0.41)$. In the antiphase minimum PRP condition entropy was $2.67(S D=1.00)$ and was 2.37 $(S D=0.73)$ in the maximum PRP condition. For the $30^{\circ}$ pattern the minimum PRP entropy was $4.30(S D=0.85)$ and $3.98(S D=1.05)$ for the maximum PRP. Note that these statistics were calculated from the entropy of all 84 experimental trials. If the $S D$ of relative phase had been calculated only 33 out of 84 trials would have been useable to estimate this statistic due to their differing significantly from a von Mises distribution.

\section{Mean Relative Phase}

The mean relative phase in the maximum in-phase PRP condition was $0.38^{\circ}(S D=$ $\left.2.85^{\circ}\right)$, minimum in-phase PRP $355.23^{\circ}\left(S D=28.44^{\circ}\right)$, maximum antiphase PRP $181.06^{\circ}\left(S D=4.19^{\circ}\right)$, minimum antiphase PRP $172.98^{\circ}\left(S D=29.45^{\circ}\right)$, maximum $30^{\circ} \mathrm{PRP} 92.74^{\circ}\left(S D=50.56^{\circ}\right)$, and minimum $30^{\circ} \mathrm{PRP} 100.73^{\circ}\left(S D=44.17^{\circ}\right)$. These values for in-phase and antiphase indicate the typical intrinsic stability of these coordination patterns. The deviation of the $30^{\circ}$ pattern toward antiphase and its greater variability indicate the typical instability of and attraction of this pattern toward antiphase due to the influence of the intrinsic dynamics landscape (Buchanan, Zihlman, Ryu, \& Wright, 2007; Fontaine, Lee, \& Swinnen, 1997; Kelso \& Zanone, 2002; Zanone \& Kelso, 1992, 1997). 


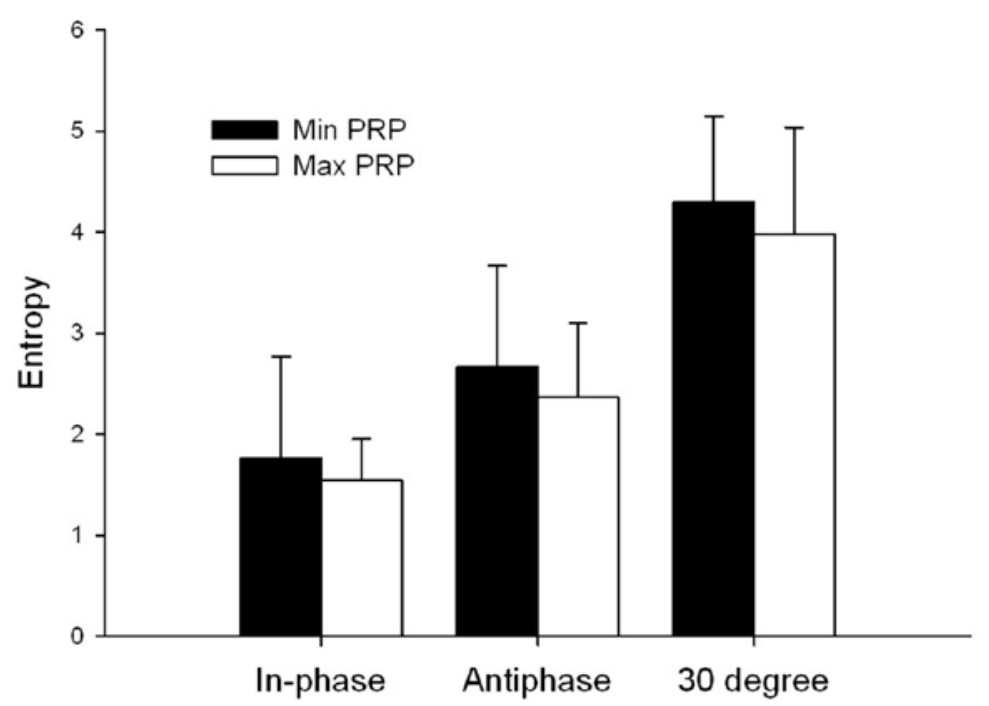

Figure 2 - Entropy of relative phase as a function of relative phase condition and effector endpoint position.

\section{Skewness and Kurtosis}

A one-sample t test of means of the circular skewness of time series distributions showed that no condition differed significantly from zero (all $p>.05$ ). See Figure 3 for a representation of these data. A one-sample t test of means of the circular kurtosis of time series distributions showed that all three relative phase patterns differed significantly (both maximum and minimum PRP in-phase conditions $p<$ .001 ; both maximum and minimum PRP antiphase conditions $p<.001 ; 30^{\circ}$ maximum PRP $p<.05)$ from the kurtosis of the von Mises distribution (zero). Only the $30^{\circ}$ minimum PRP condition did not differ significantly from the kurtosis of a von Mises distribution $(p>.05)$. The in-phase and antiphase patterns contained higher kurtosis (i.e., leptokurtic distributions) than a von Mises distribution while the $30^{\circ}$ pattern contained a lower kurtosis (i.e., platykurtic).

As shown in Figure 4 the ANOVA of relative phase kurtosis showed that significantly different levels of kurtosis occurred as a function of relative phase condition, $F(2,28)=81.23, p<.001$. Post hoc analysis showed that the in-phase kurtosis was significantly higher than the antiphase $(p<.05)$ and the $30^{\circ}(p<.001)$ relative phase conditions. The kurtosis in the antiphase condition was also significantly higher than in the $30^{\circ}$ relative phase condition $(p<.001)$. No significant effect for Endpoint (maximum and minimum PRP) occurred, $F(1,13)=0.001, p$ $>.05$, or for the Relative Phase $\times$ Endpoint interaction, $F(2,26)=0.061, p>.05$. Exemplar histograms of the relative phase distributions in each of the three relative phase conditions are shown in Figure 5. 


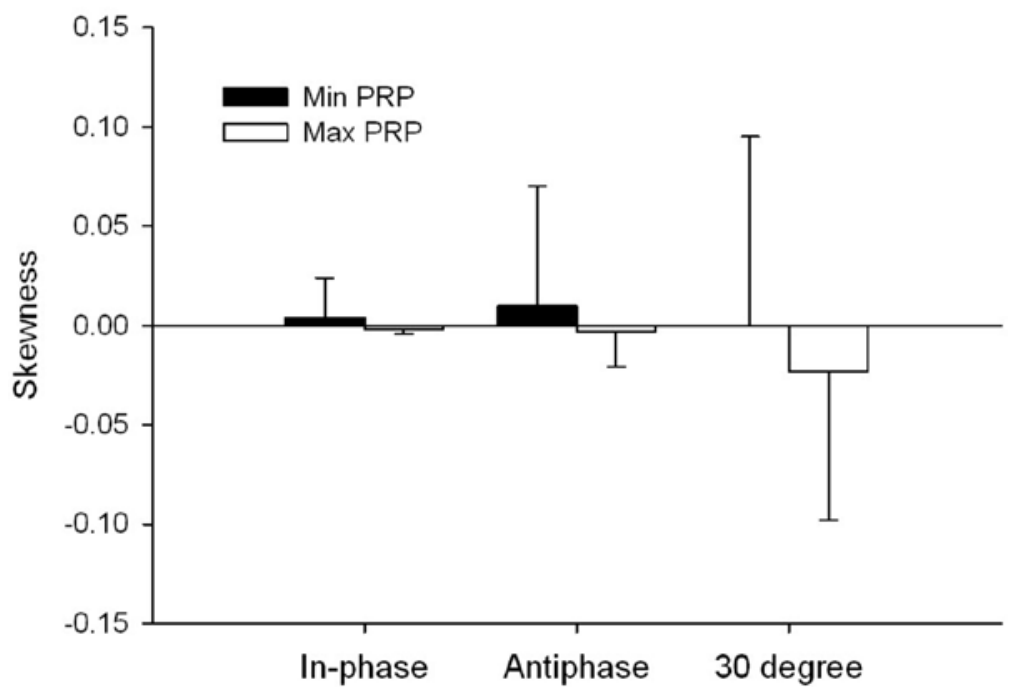

Figure 3 - Skewness of relative phase as a function of relative phase condition and effector endpoint position. MAX = endpoints of maximum elbow flexion, MIN = endpoints of maximum elbow extension.

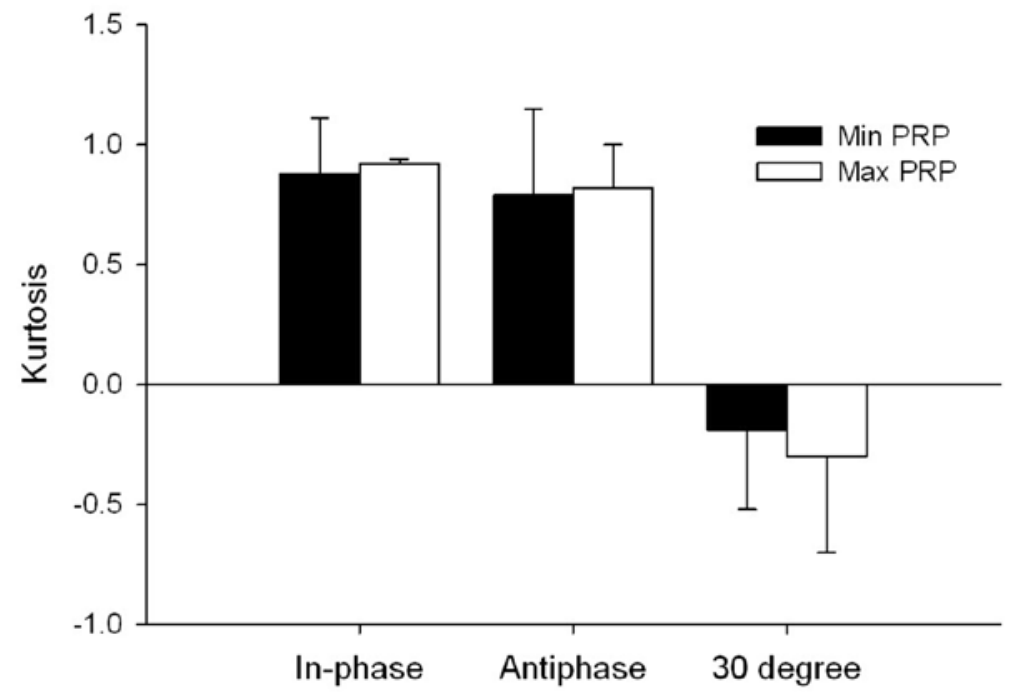

Figure 4 - Kurtosis of relative phase as a function of relative phase condition and effector endpoint position. MAX = endpoints of maximum elbow flexion; MIN = endpoints of maximum elbow extension. 

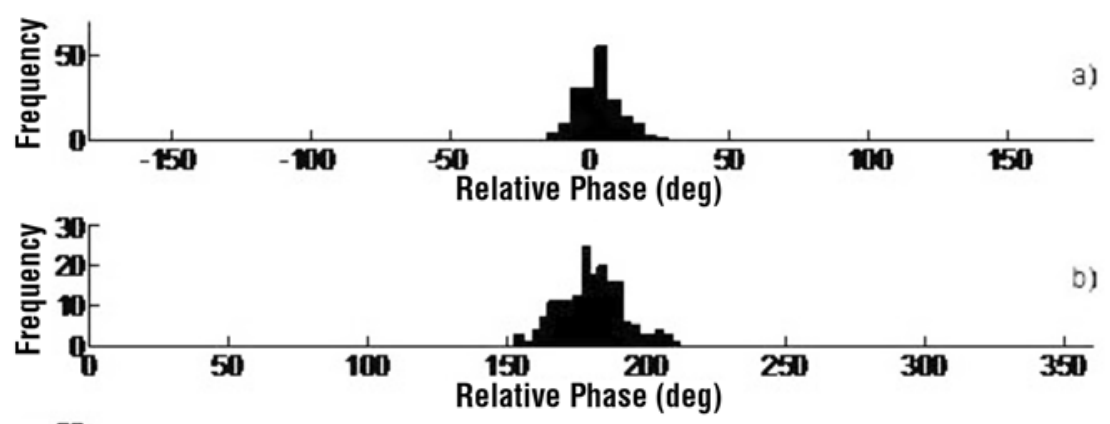

b)

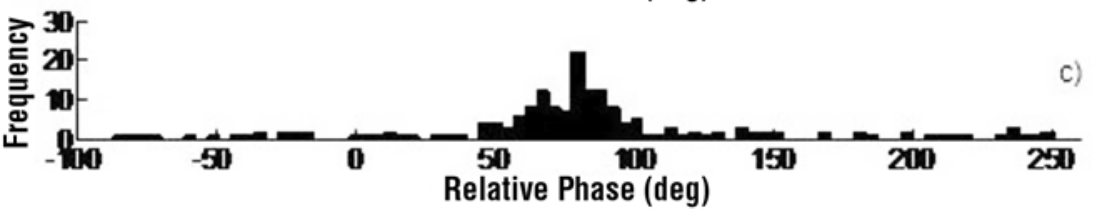

Figure 5 - Exemplar histograms of the relative phase distributions in the a) in-phase condition, b) antiphase condition, c) $30^{\circ}$ relative phase condition.

\section{Discussion}

The stability properties of bimanual coordination patterns are typically characterized through the use of the $S D$ of relative phase (Kelso, 1984; Haken et al., 1985; Post et al., 2000). The use of the $S D$ to compare across data samples depends upon the assumption of a Gaussian distribution, in the case of linear statistics, and of a von Mises distribution in circular statistics. Prior research has suggested that the relative phase of bimanual coordination is not distributed normally and that the kurtosis of relative phase distributions may change as a function of the stability properties associated with changes in movement frequency (Lee et al., 1996). The assumption of normality in relative phase distributions has not previously been formally tested.

In the present experiment it was determined that $60 \%$ of the relative phase trials differed significantly from a von Mises distribution. These trials included those that were not distinguishable from a uniform distribution as well as those that contained a modal distribution but that did not follow a von Mises distribution. Analyses of the skewness and kurtosis of time series that did not follow a von Mises distribution indicated that the in-phase and antiphase relative phase time series had significantly greater kurtosis than that of a von Mises distribution while kurtosis significantly lower than that of a von Mises distribution occurred in the $30^{\circ}$ required relative phase condition at the movement endpoints that coincided with auditory pacing tones (i.e., maximum PRP).

The intrinsically stable bimanual coordination patterns (i.e., in-phase and antiphase) possessed a significantly leptokurtic distribution while a coordination pattern considered to be intrinsically unstable (i.e., $30^{\circ}$ relative phase) possessed a platykurtic distribution. It was also found that the degree of kurtosis was a function of relative phase pattern stability, with higher kurtosis values found in more stable 
patterns (e.g., in-phase) and lower kurtosis values in less stable patterns (e.g., 30 relative phase). These findings indicate that the distributional properties of relative phase vary with the stability of a coordination pattern, with relative phase in more stable patterns being more highly concentrated around a modal value (i.e., higher kurtosis) and less stable patterns being less concentrated around a modal value (i.e., lower kurtosis).

These changes in the kurtosis of relative phase fluctuations are qualitatively consistent with the gradient properties of the behavioral attractors found in the HKB model (Haken et al., 1985). In this model, at highly stable parameter values the basin of attraction is steep and resembles the distribution found in leptokurtic data. At less stable parameter values the basin of attraction broadens and more closely resembles a less leptokurtic, or even a platykurtic, distribution. The present results show that the distributional properties of relative phase fluctuations follow these general gradient properties found in the basin of attraction as captured by the HKB model but nevertheless hold statistical limitations for inferential tests.

An implication of relative phase distribution departing from a von Mises distribution is that it may not be appropriate to use the circular $S D$ to estimate and compare the stability properties of coordination patterns in bimanual coordination. Circular statistical tests assume the presence of a von Mises distribution in data (Batschelet, 1981). However, the majority of experimental time series in the current study were determined to not follow this distribution. In light of the finding that experimental relative phase data oftentimes do not satisfy the von Mises assumption of standard circular statistics, and in the interest of a conservative approach to experimental analyses, the use of entropy-based statistical measures (e.g., information entropy; Williams, 1997) of variability might be more appropriate than the standard distributional property of $S D$. Entropy measures do not depend upon assumptions regarding the distributional properties within data and, therefore, provide a more conservative and potentially more accurate means of assessing and comparing variability, and by implication stability, within relative phase time series.

\section{References}

Batschelet, E. (1981). Circular statistics in biology. London: Academic Press.

Buchanan, J.J. Zihlman, K., Ryu, Y.U., \& Wright, D.L. (2007). Learning and transfer of a relative phase pattern and a joint amplitude ratio in a rhythmic multijoint arm movement. Journal of Motor Behavior, 39, 49-67.

Byblow, W.D., Summers, J.J., Semjen, A., Wuyts, I.J., \& Carson, R.G. (1999). Spontaneous and intentional pattern switching in a multisegmental bimanual coordination task. Motor Control, 3, 372-393.

Fisher, R.A. (1930). The moments of the distribution for normal samples of measure of departure from normality. Proceedings of the Royal Society of London. Series A, Containing Papers of a Mathematical and Physical Character, 130, 16-28.

Fontaine, R.J., Lee, T.D., \& Swinnen, S.P. (1997). Learning a new bimanual coordination pattern: reciprocal influences of intrinsic and to-be-learned patterns. Canadian Journal of Experimental Psychology, 51, 1-9.

Gilden, D.L., Thornton, T., \& Mallon, M.W. (1995). 1/f noise in human cognition. Science, 267, 1837-1839.

Haken, H. (1977). Synergetics: an introduction: nonequilibrium phase transitions and self-organization in physics, chemistry, and biology. New York, NY: Springer-Verlag. 
Haken, H. (1984). The science of structure: synergetics. New York, NY: Van Nostrand Reinhold.

Haken, H., Kelso, J.A.S., \& Bunz, H. (1985). A theoretical model of phase transitions in human hand movements. Biological Cybernetics, 51, 347-356.

Kelso, J.A.S. (1984). Phase transitions and critical behavior in human bimanual coordination. American Journal of Physiology. Regulatory, Integrative and Comparative Physiology, 15, R1000-R1004.

Kelso, J.A.S. (1995). Dynamic patterns. Cambridge, London: The MIT Press.

Kelso, J.A., \& Zanone, P.G. (2002). Coordination dynamics of learning and transfer across different effector systems. Journal of Experimental Psychology: Human Perception and Performance, 28, 776-797.

Lee, T.D., Blandin, Y., \& Proteau, L. (1996). Effects of task instructions and oscillation frequency on bimanual coordination. Psychological Research, 59, 100-106.

Molenaar, P.C., \& Newell, K.M. (2003). Direct fit of a theoretical model of phase transition in oscillatory finger motions. The British Journal of Mathematical and Statistical Psychology, 56, 199-214.

Newell, K.M., \& Hancock, P.A. (1984). Forgotten moments: a note on skewness and kurtosis as influential factors in inferences extrapolated from response distributions. Journal of Motor Behavior, 16, 320-335.

Newell, K.M., \& Slifkin, A.B. (1998). The nature of movement variability. In J.P. Piek (Ed.), Motor behavior and human skill: a multidisciplinary approach (pp. 143-160). Champaign: Human Kinetics.

Post, A.A., Peper, C.E., Daffertshofer, A., \& Beek, P.J. (2000). Relative phase dynamics in perturbed interlimb coordination: stability and stochasticity. Biological Cybernetics, 83, 443-459.

Scholz, J.P., \& Kelso, J.A. (1990). Intentional switching between patterns of bimanual coordination depends on the intrinsic dynamics of the patterns. Journal of Motor Behavior, 22, 98-124.

Schöner, G., Haken, H., \& Kelso, J.A. (1986). A stochastic theory of phase transitions in human hand movement. Biological Cybernetics, 53, 247-257.

Shannon, C.E. (1948). A mathematical theory of communication. The Bell System Technical Journal, 27, 379-423.

Slifkin, A.B., \& Newell, K.M. (1999). Noise, information transmission, and force variability. Journal of Experimental Psychology: Human Perception and Performance, 25, 837-851.

Torre, K., Delignières, D., \& Lemoine, L. (2007). 1/f (beta) fluctuations in bimanual coordination: an additional challenge for modeling. Experimental Brain Research, 183, 225-234.

Williams, G.P. (1997). Chaos theory tamed. Washington, D.C.: Joseph Henry Press.

Yamada, N. (1995). Chaotic swaying of the upright movement patterns. Human Movement Science, 14, 711-726.

Zanone, P.G., \& Kelso, J.A. (1992). Evolution of behavioral attractors with learning: nonequilibrium phase transitions. Journal of Experimental Psychology: Human Perception and Performance, 18, 403-421.

Zanone, P.G., \& Kelso, J.A. (1997). Coordination dynamics of learning and transfer: collective and component levels. Journal of Experimental Psychology: Human Perception and Performance, 23, 1454-1480. 